\title{
Photon Redshift in a Magnetic field
}

\author{
H. Pérez Rojas and E. Rodriguez Querts \\ Instituto de Cibernetica, Matematica y Fisica, \\ Calle E 309, Vedado, Ciudad Habana, Cuba.
}

(Dated: November 12, 2018)

\begin{abstract}
Previous results from the authors concerning the arising a tiny photon anomalous paramagnetic moment are also interpreted as a red-shift in analogy to the gravitational known effect. It is due to the photon interaction with the magnetized virtual electron-positron background which withdraw transverse momentum from photons and is polarization-dependent. If the photon frequency red-shift implies a change in time, a clock would go faster for increasing magnetic field intensity.
\end{abstract}

\section{INTRODUCTION}

From Newton gravity theory, the action of, say, the Sun on a planet is understood as a continuous taking of some amount of momentum, along the line joining the planet to the Sun, keeping the planet on a closed or open orbit, according to its initial energy. The bending of light rays obey to a similar effect, although General Relativity predicts twice the non relativistic shift.

In classical electrodynamics, a charged spinless particle entering in a region where a magnetic field $B$ exists, does not vary its energy. If we assume a particle with initial transverse momentum $p_{t}$, after entering in that region, Lorentz force makes it to rotate in such a way that its tangential momentum is equal to $p_{t}$. The magnetic field withdraw momentum, (which is not conserved)) but not energy. In the quantum case, the situation is quite different: the energy eigenvalues of a charged particle are dependent on $B$.

A photon propagating in vacuum in a strong magnetic field has magnetic properties due to the dependence of $\omega$ on $B$ through its interaction with the electron-positron virtual quanta, which is found by solving the photon dispersion equation giving the photon energy dependence on the self-energy tensor. This problem was studied by Shabad in 1975 1], who solved the dispersion equations in the limit of frequencies close to the first pair creation threshold, where the self-energy diverges. Paper [1] is a fundamental one, and from its results one may conclude that there is a close analogy between the bending of the light path in general relativity, a classical problem, and the similar bending of the photon path in magnetized vacuum, in the framework of quantum electrodynamics in external fields. Much more, as shown in [2], the dependence of the photon frequency on the magnetic field lead to a (polarization dependent) paramagnetic behavior in the whole interval of frequencies from zero to the first pair creation threshold, which is named the region of transparency.

But photon paramagnetism implies that $\partial \omega / \partial B<0$, and it leads to photon red-shift in a magnetic field. It is manifested due to the withdraw of photon transverse momentum by the magnetized virtual electron-positron pairs. This suggests a change in the course of time also.

\section{ELECTRON-POSITRON QUANTUM MECHANICS IN MAGNETIC FIELD}

Let us recall some details about the electron-positron quantum mechanics in a magnetic field. We assume some magnetic field defined by the field invariants $\mathcal{F}=2 B^{2}>$ $0, \mathcal{G}=0$. In a given coordinate system, a constant uniform magnetic field $\mathbf{B}$, (taken for instance, along the $x_{3}$ axis), produces a symmetry breaking of the space symmetry. For electrons and positrons $\left(e^{ \pm}\right)$physical quantities are invariant only under rotations around $x_{3}$ or displacements along it [3]. This means that the conserved quantities, i.e., those commuting with the Hamiltonian operator, are all parallel to $\mathbf{B}$, as angular momentum and spin components $J_{3}, L_{3}, s_{3}$ and the linear momentum $p_{3}$. By using units $\hbar=c=1$, the energy eigenvalues for $e^{ \pm}$are $E_{n, p_{3}}=\sqrt{p_{3}^{2}+m^{2}+e B\left(2 n+1+s_{3}\right)}$ where $s_{3}= \pm 1$ are spin eigenvalues along $x_{3}$ and $n=0,1,2$. are the Landau quantum numbers. In other words, in presence of $B$, the transverse squared energy $E_{n, p_{3}}^{2}-p_{3}^{2}$ is is given by the eigenvalues of an oscillator-like operator added to the spin $s_{3}$ operator, which quantizes in integer multiples of $e B$. The conserved quantities are those commuting with the Hamiltonian operator and we stress that the electron-positron transverse momentum squared $p_{1}^{2}+p_{2}^{2}$ is not a quantum mechanical observable and is not conserved.(The corresponding, photon momentum component $k_{\perp}^{2}$ is either not conserved). For the ground state $n=0, s=-1$, the integer is zero. Quantum states degeneracy with regard spin is expressed by a term $\alpha_{n}=2-\delta_{0 n}$, whereas degeneracy with regard to orbit's center coordinates leads to a factor $e B$, The quantity $1 / e B$ characterizes the spread of the $e^{ \pm}$spinor wavefunctions in the plane orthogonal to $B$. The magnetic moment operator $M$ is defined as the quantum average of $M=-\partial H / \partial B$, where $H$ is the Dirac Hamiltonian in the magnetic field $B$, and it is not a constant of motion. But its time-dependent terms vanish after quantum averaging and it leads to $\bar{M}=-\partial E_{n, p_{3}} / \partial B$.

Due to the explicit symmetry breaking, the four momentum operator acting on the vacuum state does not have a vanishing four-vector eigenvalue, $P_{\mu} \mid 0, B>\neq 0$. The components $P_{1,2}$ does not commute with the Hamiltonian operator $H$, and are not conserved. The $e^{ \pm}$ 
quantum vacuum energy density is given by $\Omega_{E H}=$ $-e B \sum_{n=0}^{\infty} \alpha_{n} \int d p_{3} E_{n, p_{3}}$. After removing divergences it gives the well-known Euler-Heisenberg expression $\Omega_{E H}=$ $\frac{\alpha B^{2}}{8 \pi^{2}} \int_{0}^{\infty} e^{-B_{c} x / B}\left[\frac{\operatorname{coth} x}{x}-\frac{1}{x^{2}}-\frac{1}{3}\right] \frac{d x}{x}$ which is an even function of $B$ and $B_{c}$, where $B_{c}=m^{2} / e \simeq 4.4 \times 10^{13} \mathrm{G}$ is the Schwinger critical field. The magnetized vacuum is paramagnetic $\mathcal{M}_{V}=-\partial \Omega_{E H} / \partial B>0$ and is an odd function [4] of $B$. For $B<<B_{c}$ it is $\mathcal{M}_{V}=\frac{2 \alpha}{45 \pi} \frac{B^{3}}{B_{c}^{2}}$, where $\alpha$ the fine structure constant. $\mathcal{M}_{V}$ is obviously be understood as the modulus of a vector parallel to $\mathbf{B}$.

\section{THE PHOTON RED-SHIFT FROM SHABAD'S DISPERSION EQUATIONS}

The diagonalization of the photon self-energy tensor leads to the equations [1]

$$
\Pi_{\mu \nu} a_{\nu}^{(i)}=\kappa_{i} a_{\mu}^{(i)},
$$

having three non vanishing eigenvalues and three eigenvectors for $i=1,2,3$, corresponding to three photon propagation modes. One additional eigenvector is the photon four momentum vector $k_{\nu}$ whose eigenvalue is $\kappa_{4}=0$. [1].

The dispersion equations, obtained as the zeros of the photon inverse Green function $D_{\mu \nu}^{-1}=0$, after diagonalizing the polarization operator, by using the variables $z_{1}=\omega^{2}-k_{\|}^{2}, z_{2}=k_{\perp}^{2}$, are

$$
k^{2}=\kappa_{i}\left(z_{2}, z_{1}, B\right) \quad i=1,2,3 .
$$

After solving the dispersion equations for $z_{1}$ in terms of $z_{2}$ we get

$$
\omega^{(i) 2}=|\mathbf{k}|^{2}+\mathfrak{M}^{2(i)}\left(z_{2}, B\right)
$$

We recall that for propagation orthogonal to $B$ the mode $i=2$ is polarized along $B$ and the $i=3$ is polarized perpendicular to $B$. The expansion of $\kappa_{i}$ in the limit of low frequency $\omega \ll 2 m$, low magnetic field $b=B / B_{c} \ll 1$ was done in $([2])$. By taking the first two terms in the $\kappa_{i}^{(0)}$ series expansion,

$$
\mathfrak{M}^{2(2)}=-\frac{7 \alpha z_{2}}{45 \pi}\left(b^{2}-\frac{26 b^{4}}{49}\right),
$$

and

$$
\mathfrak{M}^{2(3)}=-\frac{4 \alpha z_{2}}{45 \pi}\left(b^{2}-\frac{12 b^{4}}{7}\right) .
$$

Up to fields $B \sim 0.4 B_{c}$ one can neglect the $b^{4}$ term and write as a good approximation the dispersion equation for these modes as,

$$
\omega^{2}-k_{\|}^{2}=k_{\perp}^{2}\left(1-\frac{C^{i} \alpha b^{2}}{45 \pi}\right)
$$

where $C^{i}=7,4$ for $i=2,3$. Eq. (6) can be interpreted as the "out" dispersion equation, where the "in" one is light come equation $\omega^{2}=k_{\|}^{2}+k_{\perp}^{2}$. The effect of the magnetic field is to decrease by a factor $f(B)^{(i)}=\left(1-\frac{C^{i} \alpha b^{2}}{45 \pi}\right)$ the incoming transverse momentum, to the effective value $k_{\text {eff } \perp}^{2}=k_{\perp}^{2} f(B)^{(i)}$. From (4), (5), the frequency is red shifted when passing from a region of magnetic field $B$ to another of increased field $B+\Delta B$. In the same limit it is,

$$
\Delta \omega^{(2)}=-\frac{14 \alpha z_{2} b \Delta b}{45 \pi|\mathbf{k}|}<0
$$

and

$$
\Delta \omega^{(3)}=-\frac{8 \alpha z_{2} b \Delta b}{45 \pi|\mathbf{k}|}<0 .
$$

Thus, the red shift differs for longitudinal and transverse polarizations. The magnetic field decreases the frequency of radiation incoming to magnetized vacuum, and this decrease depends on its polarization.

To give an order of magnitude, for instance, for photons of frequency $10^{20} \mathrm{~Hz}$, and magnetic fields of order $10^{12} \mathrm{G},|\Delta \omega| \sim 10^{-6} \omega$.

In the high frequency limit $m^{2} \lesssim-z_{1} \leq 4 m^{2}, B \lesssim B_{c}$, the energy gap between successive Landau energy levels for electrons and positrons is of order close to the electron rest energy. The photon self-energy diverges for values of $-z_{1}=k_{\perp}^{\prime 2}$.

In the vicinity of the first threshold $n=n^{\prime}=0$ and by considering $k_{\perp} \neq 0$ and $k_{\|} \neq 0$, according to [1], the physical eigenwaves are described by the second and third modes, but only the second mode has a singular behavior near the threshold. The photon redshift can be written as

$$
\Delta \omega^{(2)}=\frac{\left(-4 m^{2}-z_{1}\right)\left(1+\frac{z_{2}}{2 e B}\right) \alpha m^{3} e^{-\frac{z_{2}}{2 e B}}}{\omega B_{c}\left(\left(4 m^{2}+z_{1}\right)^{3 / 2}+b \alpha m^{3} e^{-\frac{z_{2}}{2 e B}}\right)}<0,
$$

The expression (9) has a minimum near the threshold [2]. We observe that the red shift effect in the magnetic field acts as in the gravitational case for increasing fields, but in the latter, being the gravitational potential negative, when it increases, its absolute value decreases. In the magnetic field case, the increase is towards larger positive values of the field $B$.

The photon frequency shift in a magnetic field suggests a variation in the course of time. This problem requires further research, but in a simple approximation, let us assume a strongly magnetized region of space, for instance, a star. We assume that there is axial symmetry and in small regions, say, concentric equipotential shells, the magnetic field may be considered as constant in each one, and an increase means to pass from a shell to a neighbor one. Actually, if a train of waves of a fixed polarization and frequency $\omega$, containing $n$ complete oscillations during the time $T_{1}$, is sent from one shell of field $B+\Delta B$ 
to the neighbor shell having field $B$, the relation between the frequency and the interval $T_{1}$ is:

$$
T_{1}=2 \pi n / \omega
$$

The frequency of the same train of waves at the neighbor shell can be measured by dividing $2 \pi n$ between the duration of the train. The obtained number is $\omega^{\prime}>\omega$ and it means that the interval $T_{2}$ corresponding to $n$ oscillations are

$$
T_{2}=2 \pi n / \omega^{\prime}
$$

From (10), (11) it is deduced that:

$$
\frac{T_{1}-T_{2}}{T_{1}}=|\Delta \omega| / \omega^{\prime}
$$

Thus $T_{1} \simeq(1+|\Delta \omega| / \omega) T_{2}$.

That is, the clock at the first shell measured for the duration of the wave train an interval of time greater than in the second. Times goes faster for increasing $B$.

\section{ACKNOWLEDGMENTS}

The authors thank OEA-ICTP for support under Net35. One of the authors (H.P.R.) thanks Jose Helayel-Neto for hospitality at CBPF.
[1] A. E. Shabad, Ann. Phys. 90, 166 (1975).

[2] H. Pérez Rojas y E. Rodríguez Querts, Phys. Rev. D 79, 093002 (2009).

[3] M.H. Johnson, B.A. Lippmann, Phys. Rev. 76, 828 (1949).
[4] H. Pérez Rojas y E. Rodríguez Querts, Int. J. Mod. Phys A 21, 3761 (2006). 